\title{
Myocardial tissue characterization based on a polarization-sensitive optical coherence tomography system with an ultrashort pulsed laser
}

\author{
Chia-Wei Sun \\ Yih-Ming Wang \\ National Taiwan University \\ Graduate Institute of Electro-Optical Engineering \\ Taipei, Taiwan
}

\author{
Long-Sheng Lu \\ National Taiwan University \\ Graduate Institute of Pharmacology \\ Taipei, Taiwan
}

\author{
Chih-Wei Lu \\ I-Jen Hsu \\ Meng-Tsan Tsai \\ C. C. Yang \\ Yean-Woei Kiang \\ National Taiwan University \\ Graduate Institute of Electro-Optical Engineering \\ and \\ Department of Electrical Engineering \\ No. 1, Section 4, Roosevelt Road \\ Taipei, Taiwan \\ E-mail: ccy@cc.ee.ntu.edu.tw
}

\begin{abstract}
A polarization-sensitive optical coherence tomography (PSOCT) system using a femtosecond-laser as the broadband light source is implemented with the axial resolution of $5 \mu \mathrm{m}$ in free space. Through the design of path-length difference between the two polarization inputs and the modulation of one of the polarization inputs, the PSOCT images of various input and output polarization combinations can be distinguished and simultaneously collected. The PSOCT system is then used for in vitro scanning of the myocardium tissues of normal and infarcted rat hearts. The destruction of the birefringence nature of the fiber muscle in the infarcted heart can be clearly observed. () 2006 Society of Photo-Optical Instrumentation Engineers. [DOI: $10.1117 / 1.2363358]$
\end{abstract}

Keywords: medical imaging; microscopy; lasers; optical systems; polarization.

Paper 04002RRR received Jan. 5, 2004; revised manuscript received May 12, 2005 and Feb. 15, 2006; accepted for publication May 23, 2006; published online Oct. 20, 2006.

\section{Chau-Chung Wu}

National Taiwan University

Department of Primary Care Medicine and

Department of Internal Medicine

Taipei, Taiwan

\section{Introduction}

Biological tissues usually respond to various environmental cues with simultaneous functional and structural changes. The efforts to resolve material properties of living biological tissues with physical factors, also known as tissue characterization, suggested that the material properties of biological tissues are more sensitive indicators of prognosis compared with functional parameters in some pathological processes. ${ }^{1,2}$ The micro-architecture and composition of biological tissues are the major determinants of their material properties. Such information is particularly useful in the field of cardiovascular medicine. Cardiovascular death is a major health issue in developed countries. The major component of the heart is bulky myocardium, which is composed of quasi-regularly arranged muscle fibers. The average width of a normal human ventricular muscle fiber is around $23 \mu \mathrm{m} .{ }^{3}$ Most heart diseases are associated with structural changes. For example, after an

Address all correspondence to C. C. Yang, Graduate Institute of Electro-Optical Engineering, National Taiwan Univ., 1, Roosevelt Road, Section 4, Taipei, 10617 Taiwan; Tel: 8862 23657624; Fax: 886223652637 acute myocardial infarction, the damaged myocardium becomes edematous and contractured. Subsequently, the regular muscle fiber arrangement is lost within one week. In this situation, the infarcted area is replaced by randomly oriented fibrous scar within six weeks. Further pathological remodeling would result in disarrayed and hypertrophied muscle fibers in the remaining working myocardium. Any tool that characterizes detailed myocardial tissue structures can be of great value to evaluate subsequent events after a myocardial infarction for further diagnosis and treatment.

Although physiological measurements can well describe functional alterations as diseases progress, current tools for detecting the corresponding structural changes still greatly rely on post-mortem or in vitro microscopic pathological examination. This technical limitation led to the traditional concept that morphological changes occurred later than the functional alterations. However, with the development of in vivo tissue characterization techniques, information derived from structural changes seemed to be a more sensitive marker of adaption-maladaptation processes. One of the possible rea-

1083-3668/2006/11(5)/054016/7/\$22.00 @ 2006 SPIE 
sons that material properties more vividly reflect the progress of disease in the early phase is that biological tissues tend to respond to persistent perturbation to maintain functional homeostasis at the price of remodeling of tissue microarchitecture and cellular components. These subtle structural cues can be of important clinical implications. In the case of myocardial hypoperfusion, timely revascularization would rescue ischemic but viable myocardial tissue, resulting in less scar formation and better prognosis. The cardiac functional recovery relies on the amount and distribution of viable tissues and the success of revascularization. This is indeed a diagnostic challenge for all times. Noninvasive methods to detect these tissue characteristics changes include proton magnetic resonance imaging with gadolinium or dysprosium chelates (e.g., Gd-DTPA) ${ }^{4,5}$ and detection of released soluble proteins (e.g., lactate dehydrogenase, creatine kinase, troponin, and myoglobin), ${ }^{6}$ particularly during the procedure of coronary artery bypass surgery. While currently there are effective techniques for noninvasive, in vivo diagnosis of myocardial injury, there is a need for effectively monitoring the membrane disruption in exposed hearts (both in the operating room and in research). For this need, the aforementioned techniques are ineffective. ${ }^{7}$ The damaged myocardium would become edematous, contracted, and infiltrated with inflammatory cells and would lose regular alignment of muscle fibers. However, to identify most of these early structural changes, spatial resolution up to the sub-mm level is required. Such resolution is beyond the limit of all current systems. Therefore, the development of an adequate diagnostic modality that can characterize the tissue of interest with microscopic resolution is needed.

Optical coherence tomography (OCT) has proven useful for biological tissue monitoring of high resolution and fast scanning. ${ }^{8}$ With super-continuum light sources, the axial resolution of OCT has been pushed down to around, or even below, $1 \mu \mathrm{m} .^{9-12}$ Also, because of the development of rapid-scanning optical delay line (RSODL) and other techniques for fast scanning and data processing, an imaging speed as high as more than 16 frames per sec $(100 \times 50$ pixels) has been reported. ${ }^{13-15}$ OCT has been widely applied to scanning various portions of biological tissues and the diagnoses of various diseases, including urinary bladder, ${ }^{16}$ esophagus, ${ }^{17}$ central corneal epithelial, ${ }^{18}$ gastrointestinal tissue, ${ }^{19}$ atherosclerosis, ${ }^{20}$ and human mucosa. ${ }^{21}$ Recently, attention to OCT development has focused on the use of the polarization discrimination techniques for monitoring the anisotropy characteristics of biological tissues. ${ }^{22-25}$ Such a method has shown its great potential, particularly for diagnosing filamentous tissues. Arranged structures in biological tissues, in either the macroscopic or microscopic level, can reflect tissue birefringence. The anisotropy of proteins such as collagen, which builds up the extracellular matrix, leads to the birefringence nature. The molecular packing structure of the collagen fibers results in a higher index of refraction along the length of the fiber, when compared with that in the direction of their cross-section, leading to linear birefringence. Also, the myosin, which is contained in thick filaments of skeletal muscle, and elastin, which is contained in extracellular fiber, reveal anisotropy and hence contribute to the birefringence nature in myocardial tissues. Actually, birefringence can occur with anisotropic structures in other tissues, such as tendons, muscles, nerves, bones, cartilage, and teeth. In many situations, such a tissue loses its inherent birefringence characteristics due to a microstructure alteration with a certain disease.

Polarization-sensitive OCT (PSOCT) has been used for recording two-dimensional images of the change in polarization of circularly incoming light backscattered from a biological sample with birefringence. ${ }^{26,27}$ In such a system, the detected backscattered signals of the sample not only provide the information of the sample structure but also reveal sample birefringence as a function of depth. With a systematic analysis of the backscattered light from biological tissues, the StokesMuller formalism can be built based on PSOCT measurements. ${ }^{28-32}$ For easier alignment and handling, fiberbased PSOCT systems were usually used. ${ }^{33-35}$ To increase the scanning speed, we demonstrated the use of the spectral domain PSOCT scheme. ${ }^{36,37}$ Based on the Linnik microscope instrument, the full-field PSOCT was also developed to achieve micron-scale resolution with a thermal-light source. ${ }^{38,39}$ Because PSOCT enables us to detect the polarization states of backscattered signals, it can be used as a sensitive indicator of disorganization and degradation of tissues for biomedical diagnosis, including tendons and ligaments, ${ }^{40}$ cartilage, ${ }^{41,42}$ dentin and enamel, ${ }^{43,44}$ burn lesions of skin, ${ }^{45-47}$ cornea, ${ }^{48,49}$ retina, ${ }^{50}$ retinal nerve fibers, ${ }^{51}$ coronary tissue, ${ }^{52,53}$ and invasive basal cell carcinoma. ${ }^{54}$

In a PSOCT system, the simultaneous collection of OCT images of various polarization combinations can help in scanning efficiency and data consistency. Two identical light sources were simultaneously used for implementing such PSOCT image scanning. In this effort, so far the used light source is restricted to super-luminescence diodes (SLDs). Although an SLD has the advantages of stability, compactness, and portability, it usually has a relatively lower power and narrower spectral width when compared with an ultrashort pulsed solid-state laser or a super-continuum source pumped with such a laser. With an ultrashort pulsed solid-state laser, the broader spectrum leads to higher resolution and the higher output power results in higher sensitivity.

In this paper, we report the implementation of a PSOCT system and its application to diagnosing myocardium tissues. In the system, a relative delay of the pulsed signals between the two input polarization components is designed to avoid their interference. Also, modulation of signals in one of the input polarization paths is used for distinguishing the interfered signals at the detectors between the two polarization conditions. With such a setup, the OCT images of various polarization combinations can be obtained simultaneously. The PSOCT system is then applied to diagnosing the differences of myocardium tissues between the normal and infarcted rat hearts. To determine the optimal therapy for such diseases, it is important to understand the structure of abnormal myocardium tissues. Therefore, the anisotropy information from PSOCT images of such a tissue is quite helpful.

This paper is organized as follows: in Section 2, we describe the experimental setup, experimental procedures, and sample preparation. Experimental results are discussed in Section 3. Finally, discussions and conclusions are drawn in Section 4. 


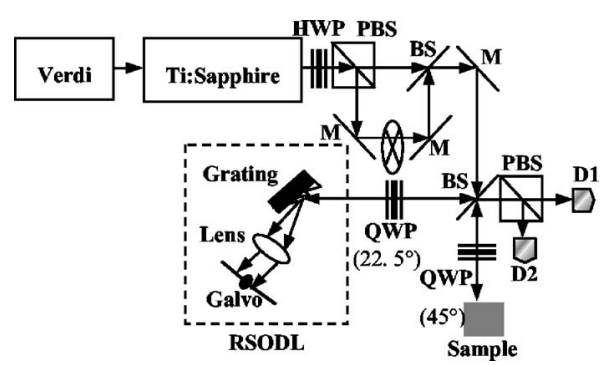

Fig. 1 Setup of the PSOCT system. PBS: polarization beam splitter; BS: beam splitter; M: mirror; C: chopper; HWP: half-wave plate; QWP: quarter-wave plate; D1 and D2: photo-detectors.

\section{Experimental Procedures and Sample Preparation}

The setup of the PSOCT system is shown in Fig. 1. It was built with a Verdi-laser-pumped mode-locked Ti: sapphire laser (Femtosource Compact Pro, FEMTOLASERS, Austria), which provided $12 \mathrm{fs}, 76-\mathrm{MHz}$ pulses with the central wavelength at $800 \mathrm{~nm}$. The full-width-at-half-maximum (FWHM) of the laser spectrum ranged from 80 to $100 \mathrm{~nm}$, depending on the mode-locking condition. Such a spectral width corresponds to the axial resolution between 2.8 and $3.5 \mu \mathrm{m}$ in free space. The output of the $\mathrm{Ti}$ : sapphire laser was horizontally polarized. It was rotated into $45^{\circ}$ linear polarization through a half-wave plate (QWPO-780-10-2-R10, CVI). Then the laser beam was split into two branches of the horizontal and vertical polarization with different path lengths by a polarization beam splitter (PBSH-670-980-100, CVI). The path-length difference meant to avoid the interference of signals between the two polarization inputs. A chopper was used for $4-\mathrm{kHz}$ modulation to differentiate the signals of the vertical polarization input from the other. The two input beams were merged with a nonpolarization beam splitter (50/50 splitting ratio; BBS650-1100-1025-45, CVI). About $600 \mathrm{~mW}$ of the average power of laser pulses was applied to the free-space Michelson interferometer. Optical signals in the reference arm passed through a zeroth-order quarter-wave plate (QWPO-780-10-4$\mathrm{R} 10$, CVI), set at $22.5^{\circ}$ with respect to the horizontal direction, such that the signal polarization was rotated by $45^{\circ}$ on reflection from the RSODL. In the reference arm, after returning from the RSODL and doubly passing through the quarterwave plate, the horizontal polarization component of the incident light was converted into the $45^{\circ}$ polarization while the vertical polarization component of the incident light was converted into the $-45^{\circ}$ direction. The RSODL in the reference arm was built for phase modulation and depth scanning. The diffraction efficiencies of the used grating (400 lines/mm, Richardson Grating Laboratory) in the RSODL are $73 \%$ and $77 \%$ for the horizontal and vertical polarization components, respectively. Such a difference does not affect the operation of the PSOCT system. For dispersion compensation of the OCT system, we adjusted the separation between the diffractive grating and the lens in the used RSODL. ${ }^{55}$ Another quarterwave plate, set at $45^{\circ}$ with respect to the horizontal direction, was placed in the sample arm such that circularly polarized light was incident upon the sample. The polarization states at the sample surface of the two incident polarization directions were right- and left-hand circular polarization, respectively.

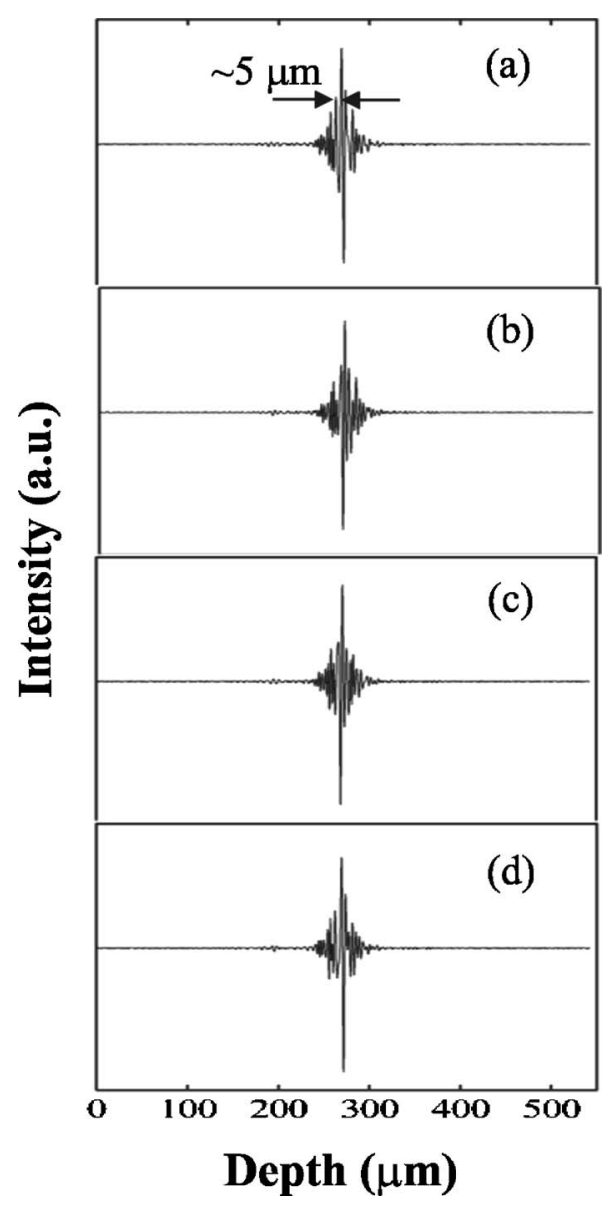

Fig. 2 Interference fringe patterns from surface reflection with parts (a)-(d) for the input and output polarization combinations $\mathrm{HH}, \mathrm{HV}$, $\mathrm{VH}$, and $\mathrm{VV}$, respectively.

The reference beam combined with the backscattered sample beam through the nonpolarization beam splitter. A polarization beam splitter was used before optical signal detection for output polarization discrimination. The two polarization outputs of interfered signals were detected by two photodetectors (model 2001, New Focus), D1 and D2, respectively. The signals were then processed with two bandpass filters (FV-665, NF Electronic Instruments) to extract the OCT images of various polarization combinations. Because of the use of the chopper, the central frequency of the bandpass filter for the vertical polarization input was shifted by $4 \mathrm{kHz}$ from that for the other polarization. The transverse resolution of the PSOCT system was about $10 \mu \mathrm{m}$. The sensitivity was estimated to be about $80 \mathrm{~dB}$. It can be improved if a more stable laser source is used. The focused laser-beam diameter on the tissue samples was around $3.5 \mathrm{~mm}$. The real power applied to the samples was only $5 \mathrm{~mW}$, corresponding to $52 \mathrm{~mW} / \mathrm{cm}^{2}$ in average power density. It should be safe for in vivo measurements with such a power density.

The four parts of Fig. 2 show the interference fringe patterns of the four polarization combinations recorded with the reflection from a glass surface. Parts (a)-(d) correspond to the cases of $\mathrm{HH}, \mathrm{HV}, \mathrm{VH}$, and VV, respectively. The first (second) letter represents the input (output) polarization direction. $\mathrm{H}$ and $\mathrm{V}$ mean the horizontal and vertical directions, respec- 

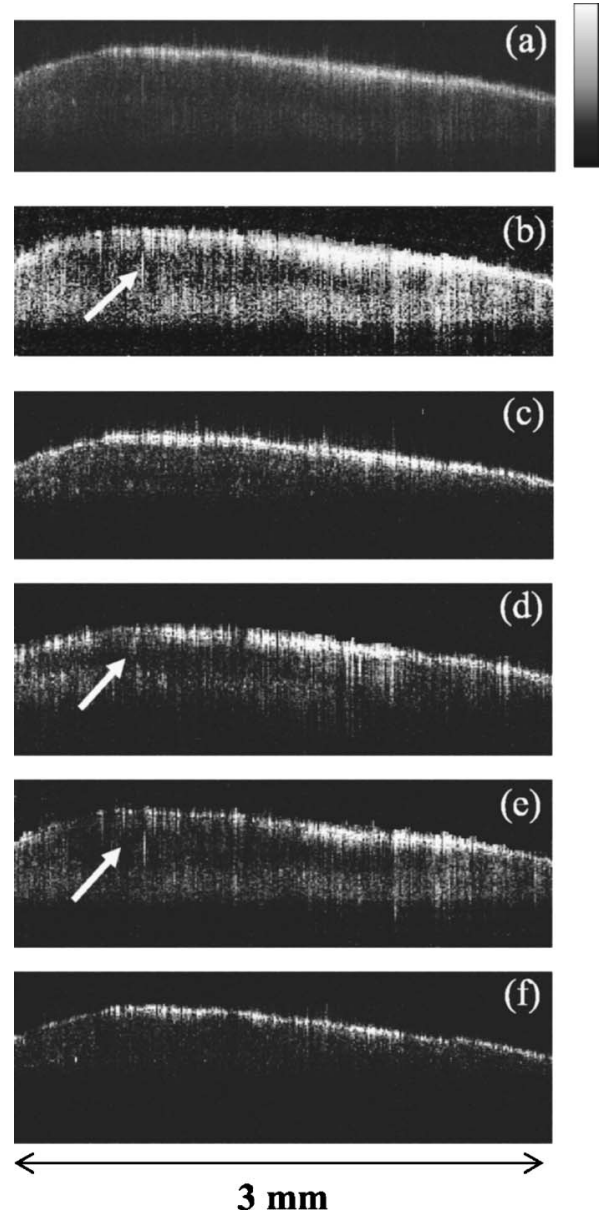

Fig. 3 PSOCT images of the myocardium tissue of the normal rat heart with parts (a)-(f) for the structure, phase retardation, and input and output polarization combinations $\mathrm{HH}, \mathrm{HV}$, VH, and $\mathrm{V}$, respectively.

tively. The fringe envelopes of the four polarization combinations look similar and all have the FWHM around $5 \mu \mathrm{m}$, corresponding to a resolution of about $3.57 \mu \mathrm{m}$ in biological tissues. With this result, we believe that the dispersion mismatch is essentially compensated for in this free-space PSOCT system.

The PSOCT system was then used for scanning normal and abnormal myocardium tissues of rats. Sample preparation was conducted in accordance with the R.O.C. Animal Protection Law (Scientific Application of Animals), 1998. After a bolus of intraperitoneal pentobarbital $(50 \mathrm{mg} / \mathrm{kg})$, the left anterior descending coronary artery of an adult WKY rat was ligated. The ligation was kept for eight weeks for the infarction to become mature. A sham operation was performed on the other rat as on the normal sample for comparison. To harvest the hearts, a lethal dose of sodium pentobarbital was administered. The animals were retrogradely perfused with heparinized PBS (10 units/ml) for $15 \mathrm{~min}$. The hearts were then quickly removed and fixed onto the sample stage of the PSOCT system. The midventricular myocardium around the left anterior descending artery was scanned.

\section{Experimental Results}

Parts (a)-(f) of Fig. 3 show the PSOCT images of normal myocardial tissue of a rat for the structure, phase retardation, $\mathrm{HH}, \mathrm{HV}, \mathrm{VH}$, and VV polarization combinations, respectively. In the images, the sporadic vertical line structures are attributed to laser instability. As mentioned before, the birefringence characteristics of myocardial tissue originate from its anisotropic fiber architecture. ${ }^{56}$ The phase retardation between 0 to $\pi$, as shown in Fig. 3(b), indicates the polarization evolution in the myocardium. In a tissue sample of isotropic scattering, the nonpolarized backscattered light should lead to a continuous-phase retardation distribution in image. In the four polarization-resolved images [Figs. 3(c)-3(f)], a smooth epicardial contour with a layered pattern can be seen. A middle layer of a weak signal can be clearly seen in the $\mathrm{HV}$ and $\mathrm{VH}$ components of the PSOCT images of the normal heart, as indicated with the white arrows in Figs. 3(d) and 3(e). The dark layer is supposed to originate from the variation of fiber muscle orientation along the sample depth. The orientation of the muscle bundles rotates by approximately $180^{\circ}$ from epicardium to endocardium when examined in a midventricular cross-section. Hence, the muscle fibers in the middle part of the ventricular wall are approximately perpendicular to those in the epi- and endocardium. The dark layer in the OCT images corresponded to this topography. Because the structure image [Fig. 3(a)] shows quite a continuous distribution, we can conclude that the HV and VH images [Figs. 3(d) and 3(e)] reflect real tissue birefringence.

On the other hand, the PSOCT images acquired from an infarcted heart displayed a significant difference. Figures 4(a)-4(f) show the PSOCT images of the infarcted heart for the structure, phase retardation, and $\mathrm{HH}, \mathrm{HV}, \mathrm{VH}$, and $\mathrm{VV}$ polarization combinations, respectively. Here, one can see that the layered patterns in Fig. 3 disappear. In the macroscopic observation, the epicardial surface of the scanned area was contracted and uneven. The scarred ventricular wall was also less elastic, compared with the rest of the heart. The PSOCT images show an exaggerated border in the epicardial surface. In addition, the patchy pattern of the PSOCT images suggests the presence of marked heterogeneities in the sample. In the sample, the repair process was almost complete, and the orderly arranged muscle structure was replaced with randomly oriented collagen bundles. ${ }^{31}$ Hence, this corresponds well to our finding that in the scar region of the infarcted heart, the patchy pattern replaced the layered pattern in the PSOCT images, as shown in Fig. 4.

\section{Discussions and Conclusions}

We have demonstrated that the PSOCT technique could differentiate scars from normal myocardium in vitro. Such a technique has the potential for providing real-time information about myocardial viability, which can assist the decision of revascularization during percutaneous coronary intervention. Such information is particularly important in the cases of delayed presentation of a myocardial infarction (postinfarction angina), previously undetected multivessel diseases, and multiple stenosis with occluded proximal segments. Currently, these issues are addressed with the functional data such as intracoronary flow velocity and pressure. The PSOCT imaging provides an alternative source of information based on myocardial morphology. The possible application of this technique would not and should not be 

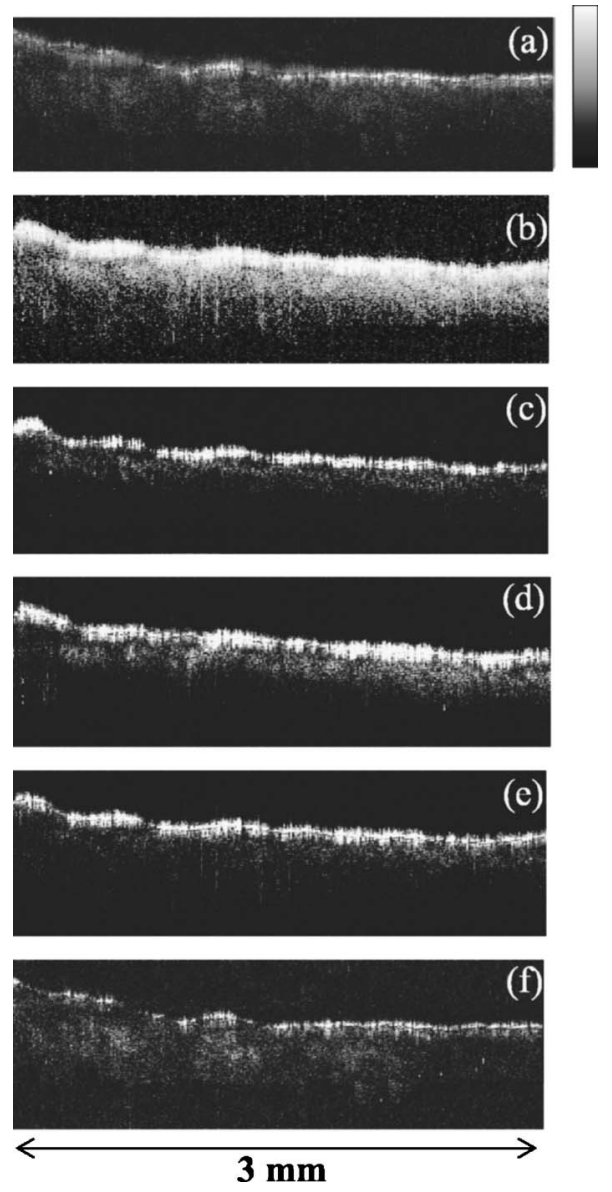

Fig. 4 PSOCT images of the myocardium tissue of the infarcted rat heart with parts (a)-(f) for the structure, phase retardation, and input and output polarization combinations $\mathrm{HH}, \mathrm{HV}, \mathrm{VH}$, and $\mathrm{VV}$, respectively.

limited to the evaluation of myocardial viability. Since this technique is sensitive to interstitial collagen deposition, early diagnosis of hereditary cardiomyopathy, transplant rejection, and cardiac fibrosis would also be potential fields of application.

As we pointed out earlier, the major strength of this technique is the high spatial resolution in comparison to PET, CT, MR, and dobutamine stress echocardiography (DSE). Another advantage of this technique is its capability to determine the supply artery of injured myocardium without the assumption of normal segmental supply pattern. Currently, the available imaging methods, including PET, DSE, and thallium scan, all detect possible infarct-related artery with the principle of segmental supply of coronary arteries. However, these methods do not work well in the cases of watershed, extensive collateral formation, variants of coronary supply pattern, and poor flow. Approaching the proximity of injured myocardium and detecting its viability in the stenosed artery with PSOCT can help in solving such a problem.

In summary, we have implemented a PSOCT system with a femtosecond laser as the broadband light source. The axial resolution reached $5 \mu \mathrm{m}$ in free space. With the design of a path-length difference between the two polarization inputs and the modulation of one of the polarization inputs, the
PSOCT images of various input and output polarization combinations could be well distinguished and simultaneously collected. The PSOCT system was then successfully used for in vitro characterization of normal and infarcted myocardial tissues. To avoid the huge calculations with the Stokes-Mueller formalism in a PSOCT system, we used the phase-retardation images to describe the birefringence effect of myocardium. Therefore, this system can provide real-time information about the changes of the anisotropic structures in heart tissues. Although only in vitro experiments were reported in this paper, this PSOCT system could offer a potential diagnostic modality for in vivo tissue characterization. Future work includes the construction of a fiber-based PSOCT system of higher stability for in vivo diagnosis of abnormal myocardium tissues. ${ }^{55,56}$ In this new PSOCT system, a light source with the central wavelength around $1300 \mathrm{~nm}$ will be used for deeper tissue penetration. Also, the implementation with a catheter and/or endoscopic system will be investigated in future research.

\section{Acknowledgment}

This research was supported by the National Health Research Institute, The Republic of China, under Grant NHRI-EX949220EI.

\section{References}

1. V. Giglio, V. Pasceri, L. Messano, F. Mangiola, L. Pasquini, A. Dello Russo, A. Damiani, M. Mirabella, G. Galluzzi, P. Tonali, and E. Ricci, "Ultrasound tissue characterization detects preclinical myocardial structural changes in children affected by Duchenne muscular dystrophy," J. Am. Coll. Cardiol. 42, 309-316 (2003).

2. C. C. Wu, Y. B. Liu, L. C. Lin, Y. L. Ho, C. S. Liau, and Y. T. Lee, "Ultrasonic tissue characterization with integrated backscatter during isotropic stimulation," Ultrasound Med. Biol. 26, 1413-1420 (2000).

3. O. M. Hess, J. Schneider, H. Nonogi, J. D. Carroll, K. Schneider, M. Turina, and H. P. Krayenbuehl, "Myocardial structure in patients with exercise-induced ischemia," Circulation 77, 967-977 (1988).

4. S. M. Eleff, I. J. McLennan, G. K. Hart, Y. Maruki, R. J. Traystman, and R. C. Koehler, "Shift reagent enhanced concurrent $23 \mathrm{Na}$ and $1 \mathrm{H}$ magnetic resonance spectroscopic studies of transcellular sodium distribution in the dog brain in vivo," Magn. Reson. Med. 30, 11-17 (1993).

5. M. Saeed, "New concepts in characterization of ischemically injured myocardium by MRI," Exp. Biol. Med. 226, 367-376 (2001).

6. R. N. Johnson, N. L. Sammel, and R. M. Norris, "Depletion of myocardial creatine kinase, lactate dehydrogenase, myoglobin and K1 after coronary artery ligation in dogs," Cardiovasc. Res. 15, 529-537 (1981).

7. S. P. Nighswander-Rempel, R. A. Shaw, B. Kuzio, and V. V. Kupriyanov, "Detection of myocardial cell damage in isolated rathearts with near-infrared spectroscopy," J. Biomed. Opt. 9, 779-787 (2004).

8. D. Huang, E. A. Swanson, C. P. Lin, J. S. Schuman, W. G. Stinson, W. Chang, M. R. Hee, T. Flotte, K. Gregory, C. A. Puliafito, and J. G. Fugimoto, "Optical coherence tomography," Science 254, 1178-1181 (1991).

9. W. Drexler, U. Morgner, F. X. Kärtner, C. Pitris, S. A. Boppart, X. D. Li, E. P. Ippen, and J. G. Fujimoto, "In vivo ultrahigh-resolution optical coherence tomography," Opt. Lett. 24, 1221-1223 (1999).

10. B. Povazay, K. Bizheva, A. Unterhuber, B. Hermann, H. Sattmann, A. F. Fercher, and W. Drexler, "Submicrometer axial resolution optical coherence tomography," Opt. Lett. 27, 1800-1802 (2002).

11. K. Bizheva, B. Považay, B. Hermann, H. Sattmann, W. Drexler, M. Mei, R. Holzwarth, T. Hoelzenbein, V. Wacheck, and H. Pehamberger, "Compact, broad-bandwidth fiber laser for sub-2- $\mu \mathrm{m}$ axial resolution optical coherence tomography in the 1300-nm wavelength region," Opt. Lett. 28, 707-709 (2003).

12. Y. Wang, Y. Zhao, J. S. Nelson, Z. Chen, and R. S. Windeler, "Ultrahigh-resolution optical coherence tomography by broadband continuum generation from a photonic crystal fiber," Opt. Lett. 28, 
182-184 (2003).

13. Z. Ding, Y. Zhao, H. Ren, J. S. Nelson, and Z. Chen, "Real-time phase-resolved optical coherence tomography and optical Doppler tomography," Opt. Express 10, 236-245 (2002).

14. V. Westphal, S. Yazdanfar, A. M. Rollins, and J. A. Izatt, "Real-time, high velocity-resolution color Doppler optical coherence tomography," Opt. Lett. 27, 34-36 (2002).

15. M. J. Cobb, Xiumei Liu, and Xingde Li, "Continuous focus tracking for real-time optical coherence tomography," Opt. Lett. 30, 16801682 (2005).

16. T. Q. Xie, M. L. Zeidel, and Y. T. Pan, "Detection of tumorigenesis in urinary bladder with optical coherence tomography: Optical characterization of morphological changes," Opt. Express 10, 1431-1443 (2002).

17. R. V. Kuranov, V. V. Sapozhnikova, I. V. Turchin, E. V. Zagainova, V. M. Gelikonov, V. A. Kamensky, L. B. Snopova, and N. N. Prodanetz, "Complementary use of cross-polarization and standard OCT for differential diagnosis of pathological tissues," Opt. Express 10, 707-713 (2002).

18. J. Wang, J. Thomas, I. Cox, and A. Rollins, "Noncontact measurements of central corneal epithelial and flap thickness after laser in situ keratomileusis," Invest. Ophthalmol. Visual Sci. 45, 1812-1816 (2004).

19. A. M. Rollins, R. Ung-Arunyawee, A. Chak, C. K. Wong, K. Kobayashi, M. V. Sivak, and J. A. Izatt, "Real-time in vivo imaging of human gastrointestinal ultrastructure by use of endoscopic optical coherence tomography with a novel efficient interferometer design," Opt. Lett. 24, 1358-1360 (1999).

20. M. E. Brezinski, G. J. Tearney, B. E. Bouma, J. A. Izatt, M. R. Hee, E. A. Swanson, J. F. Southern, and J. G. Fujimoto, "Optical coherence tomography for optical biopsy," Circulation 93, 1206-1213 (1996).

21. A. M. Sergeev, V. M. Gelikonov, G. V. Gelikonov, F. I. Feldchtein, R. V. Kuranov, N. D. Gladkova, N. M. Shakhova, L. B. Snopova, A. V. Shakov, I. A. Kuznetzova, A. N. Denisenko, V. V. Pochinko, Y. P. Chumakov, and O. S. Streltzova, "In vivo endoscopic OCT imaging of precancer and cancer states of human mucosa," Opt. Express 1, 432-440 (1997)

22. M. J. Everett, K. Schoenenberger, B. W. Colston Jr., and L. B. Da Silva, "Birefringence characterization of biological tissue by use of optical coherence tomography," Opt. Lett. 23, 228-230 (1998).

23. S. Guo, J. Zhang, L. Wang, J. S. Nelson, and Z. Chen, "Depthresolved birefringence and differential optical axis orientation measurements with fiber-based polarization-sensitive optical coherence tomography," Opt. Lett. 29, 2025-2027 (2004).

24. M. Pircher, E. Goetzinger, R. Leitgeb, and C. K. Hitzenberger, "Three-dimensional polarization sensitive OCT of human skin in vivo," Opt. Express 12, 3236-3244 (2004).

25. J. Zhang, S. Guo, W. Jung, J. S. Nelson, and Z. Chen, "Determination of birefringence and absolute optic axis orientation using polarization-sensitive optical coherence tomography with PM fibers," Opt. Express 11, 3262-3270 (2003).

26. M. R. Hee, D. Huang, E. A. Swanson, and J. G. Fujimoto, "Polarization-sensitive low-coherence reflectometer for birefringence characterization and ranging," J. Opt. Soc. Am. B 9, 903-908 (1992).

27. J. F. de Boer, T. E. Milner, M. J. van Gemert, and J. S. Nelson, "Two-dimensional birefringence imaging in biological tissue by PSOCT," Opt. Lett. 22, 934-936 (1997).

28. J. F. de Boer, T. E. Milner, and J. S. Nelson, "Determination of the depth-resolved Stokes parameters of light backscattered from turbid media by use of polarization-sensitive optical coherence tomography," Opt. Lett. 24, 300-302 (1999).

29. S. Jiao and L. V. Wang, "Two-dimensional depth-resolved Mueller matrix of biological tissue measured with double-beam polarizationsensitive optical coherence tomography," Opt. Lett. 27, 101-103 (2002).

30. H. Ren, Z. Ding, Y. Zhao, J. Miao, J. S. Nelson, and Z. Chen, "Phaseresolved functional optical coherence tomography: Simultaneous imaging of in situ tissue structure, blood flow velocity, standard deviation, birefringence, and Stokes vectors in human skin," Opt. Lett. 27, 1702-1704 (2002).

31. M. Todorović, S. Jiao, and L. V. Wang, "Determination of local polarization properties of biological samples in the presence of diattenuation by use of Mueller optical coherence tomography," Opt. Lett. 29, 2402-2404 (2004).
32. S. Jiao and L. V. Wang, "Jones-matrix imaging of biological tissues with quadruple-channel optical coherence tomography," J. Biomed. Opt. 7, 350-358 (2002).

33. C. E. Saxer, J. F. de Boer, B. H. Park, Y. Zhao, Z. Chen, and J. S. Nelson, "High-speed fiber-based polarization-sensitive optical coherence tomography of in vivo human skin," Opt. Lett. 25, 1355-1357 (2000).

34. M. C. Pierce, B. H. Park, B. Cense, and J. F. de Boer, "Simultaneous intensity, birefringence, and flow measurements with high-speed fiber-based optical coherence tomography," Opt. Lett. 27, 1534-1536 (2002).

35. B. H. Park, M. C. Pierce, B. Cense, and J. F. de Boer, "Jones matrix analysis for a polarization-sensitive optical coherence tomography system using fiber-optic components," Opt. Lett. 29, 2512-2514 (2004).

36. Y. Yasuno, S. Makita, Y. Sutoh, M. Itoh, and T. Yatagai, "Birefringence imaging of human skin by polarization-sensitive spectral interferometric optical coherence tomography," Opt. Lett. 27, 1803-1805 (2002)

37. Y. Yasuno, S. Makita, T. Endo, M. Itoh, T. Yatagai, M. Takahashi, C. Katada, and M. Mutoh, "Polarization-sensitive complex Fourier domain optical coherence tomography for Jones matrix imaging of biological samples," Appl. Phys. Lett. 85, 3023-3025 (2004).

38. J. Moreau, V. Loriette, and A.-C. Boccara, "Full-field birefringence imaging by thermal-light polarization-sensitive optical coherence tomography. I. Theory," Appl. Opt. 42, 3800-3810 (2003).

39. J. Moreau, V. Loriette, and A.-C. Boccara, "Full-field birefringence imaging by thermal-light polarization-sensitive optical coherence tomography. II. Instrument and results,” Appl. Opt. 42, 3811-3818 (2003).

40. S. D. Martin, N. A. Patel, S. B. Adams, M. J. Roberts, S. Plummer, D. L. Stamper, J. G. Fujimoto, and M. E. Brezinski, "New technology for assessing microstructural components of tendons and ligaments," Int. Orthop. 27, 184-189 (2003).

41. W. Drexler, D. Stamper, C. Jesser, X. D. Li, C. Pitris, and K. Saunders, "Correlation of collagen organization with polarization sensitive imaging in cartilage: Implications for osteoarthritis," J. Rheumatol. 28, 1311-1318 (2001).

42. X. Li, S. D. Martin, C. Pitris, R. Ghanta, D. L. Stamper, M. Harman, J. G. Fujimoto, and M. E. Brezinski, "High-resolution optical coherence tomography imaging of osteoarthritic cartilage during open knee surgery," Arthritis Res. Ther. 7, R318-R323 (2005).

43. A. Baumgartner, S. Dichtl, C. K. Hitzenberger, H.Sattman, B.Robl, A.Moritz, A. F.Fercher, and W.Sperr, "Polarization sensitive optical coherence tomography of dental structures," Caries Res. 34, 59-69 (2000).

44. Y. Chen, L. Otis, D. Piao, and Q. Zhu, "Characterization of dentin, enamel, and carious lesions by a polarization-sensitive optical coherence tomography system," Appl. Opt. 44, 2041-2048 (2005).

45. B. H. Park, C. Saxer, S. M. Srinivas, J. Stuart Nelson, and J. F. de Boer, "In vivo burn depth determination by high-speed fiber-based polarization sensitive optical coherence tomography," J. Biomed. Opt. 6, 474-479 (2001).

46. S. Jiao, W. Yu, G. Stoica, and L. V. Wang, "Contrast mechanisms in polarization-sensitive Mueller-matrix optical coherence tomography and application in burn imaging," Appl. Opt. 42, 5191-5197 (2003).

47. S. M. Srinivas, J. F. de Boer, H. Park, K. Keikhanzadeh, H. L. Huang, J. Zhang, W. Q. Jung, Z. Chen, and J. S. Nelson, "Determination of burn depth by polarization-sensitive optical coherence tomography," J. Biomed. Opt. 9, 207-212 (2004).

48. M. G. Ducros, J. F. de Boer, H.-E. Huang, L. C. Chao, Z. Chen, J. S. Nelson, T. E. Milner, and H. G. Rylander III, "Polarization sensitive optical coherence tomography of the rabbit eye," IEEE J. Sel. Top. Quantum Electron. 5, 1159-1167 (1999).

49. E. Götzinger, M. Pircher, M. Sticker, A. F. Fercher, and C. K. Hitzenberger, "Measurement and imaging of birefringent properties of the human cornea with phase-resolved, polarization-sensitive optical coherence tomography," J. Biomed. Opt. 9, 94-102 (2004).

50. M. G. Ducros, J. D. Marsack, H. G. Rylander III, S. L. Thomsen, and T. E. Milner, "Primate retina imaging with polarization-sensitive optical coherence tomography," J. Opt. Soc. Am. A 18, 2945-2956 (2001).

51. B. Cense, T. C. Chen, B. H. Park, M. C. Pierce, and J. F. de Boer, "In vivo depth-resolved birefringence measurements of the human retinal nerve fiber layer by polarization-sensitive optical coherence tomog- 
raphy," Opt. Lett. 27, 1610-1612 (2002).

52. S. D. Giattina, B. K. Courtney, P. R. Herz, M. Harman, S. Shortkroff, L. Debra, D. L. Stamper, B. Liu, J. G. Fujimoto, and M. E. Brezinski, "Measurement of coronary plaque collagen with polarization sensitive optical coherence tomography (PS-OCT)," Cardiovascular Revascularization Therapies Mtg. Washington, D.C., p. 609 (2005).

53. M. C. Pierce, M. Shishkov, B. H. Park, N. A. Nassif, B. E. Bouma, G. J. Tearney, and J. F. de Boer, "Effects of sample arm motion in endoscopic polarization-sensitive optical coherence tomography," Opt. Express 13, 5739-5749 (2005).
54. J. Strasswimmer, M. C. Pierce, B. H. Park, V. Neel, and J. F. de Boer, "Polarization-sensitive optical coherence tomography of invasive basal cell carcinoma," J. Biomed. Opt. 9, 292-298 (2004).

55. W. K. Niblack, J. O. Schenk, B. Liu, and M. E. Brezinski, "Dispersion in a grating-based optical delay line for optical coherence tomography," Appl. Opt. 42, 4115-4118 (2003).

56. C. W. Sun, L. S. Lu, C. C. Yang, Y. W. Kiang, and M. J. Su, "Myocardial tissue characterization based on the time-resolved StokesMueller formalism," Opt. Express 10, 1347-1353 (2002). 